\title{
ION DIFFUSION MODELLING OF FRICKE-AGAROSE DOSEMETER GELS
}

\author{
F. de Pasquale ${ }^{1}$, P. Barone ${ }^{1}$, G. Sebastiani ${ }^{1}$, F. d’Errico ${ }^{2}$, E. Egger ${ }^{3}$, A. M. Luciani ${ }^{4}$, M. Pacilio ${ }^{4}$, \\ L. Guidoni ${ }^{4}$ and V. Viti, ${ }^{4}$, \\ ${ }^{1}$ Istituto per le Applicazioni del Calcolo, CNR, viale del Policlinico 137, 00161 Rome, Italy \\ ${ }^{2}$ Yale University School of Medicine, New Haven, CT, USA \\ ${ }^{3}$ Paul Scherrer Institut, Villigen, Switzerland \\ ${ }^{4}$ Dipartimento di Tecnologie e Salute, Istituto Superiore di Sanità and INFN-Sez.Roma 1, \\ viale Regina Elena 299, 00161 Rome, Italy
}

\begin{abstract}
In Fricke-agarose gels, an accurate determination of the spatial dose distribution is hindered by the diffusion of ferric ions. In this work, a model was developed to describe the diffusion process within gel samples of finite length and, thus, permit the reconstruction of the initial spatial distribution of the ferric ions. The temporal evolution of the ion concentration as a function of the initial concentration is derived by solving Fick's second law of diffusion in two dimensions with boundary reflections. The model was applied to magnetic resonance imaging data acquired at high spatial resolution ( $0.3 \mathrm{~mm})$ and was found to describe accurately the observed diffusion effects.
\end{abstract}

\section{INTRODUCTION}

The diffusion of ferric ions is one of the main problems that limits the applicability of Fricke-agarose gels in radiation dosimetry. A significant degradation of the recorded dose distributions can occur since the timescale of the diffusion process is comparable with the duration of the experimental procedures. The latter include irradiation, transfer of irradiated samples to the imaging system, MR system set-up and image acquisition. In some cases, an effective solution to the diffusion problem can be provided by the use of ferric ion chelators, which contrast ion mobility. In particular, the use of Xylenol Orange, proposed by Appleby and Leghrouz $^{(1)}$, drastically reduces the diffusion effects while causing a limited reduction in sensitivity of the Fricke-gel system. This compound specifically binds to ferric ions, thus, slowing down their diffusion. However, when strong concentration gradients are present, diffusion effects cannot be neglected and a reconstruction of the initial ion distribution at the end of irradiation is necessary. This can be achieved by using an appropriate model for diffusion ${ }^{(2)}$, which depends on the unknown diffusion coefficient. In this work, we propose a simple method to estimate this coefficient.

\section{MATERIALS AND METHODS}

Fricke-agarose gels were prepared in cylindrical Perspex holders according to previously published methods ${ }^{(3)}$. The final concentrations were: $1.5 \mathrm{mM}$ ferrous ammonium sulphate, $1 \mathrm{mM}$ sodium chloride,

*Corresponding author: viti@iss.it
$50 \mathrm{mM}$ sulphuric acid and 1\% agarose by weight. Hereafter, this sample will be referred to as 'Noxyl'. Samples containing Xylenol Orange were prepared with the same procedure except for adding Fricke solution containing the chelator agent. The final ferrous ammonium sulphate concentration was $1 \mathrm{mM}$ and the final Xylenol Orange concentration was $0.25 \mathrm{mM}$. These gels will be referred to as 'Xyl'. The gel samples were always kept at room temperature and irradiated between 20 and $28 \mathrm{~h}$ after preparation.

A first set of samples had a diameter of $45 \mathrm{~mm}$ and a height of $40 \mathrm{~mm}$ (Figure 1). These were coaxially irradiated at the Paul Scherrer Institut in Villigen, Switzerland, with a $62 \mathrm{MeV}$ proton beam, which was modulated to yield a spread-out Bragg peak. Reference dosimetry was performed using a parallel plate

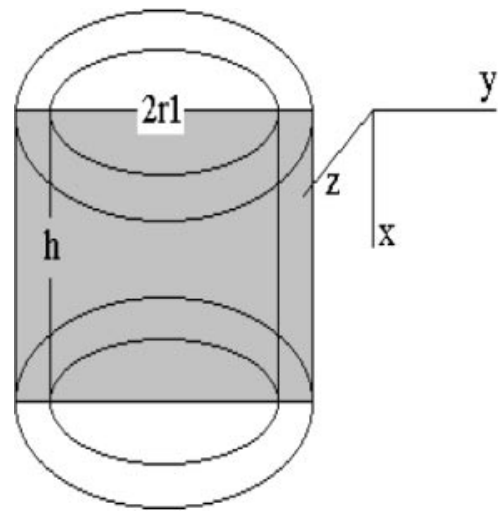

Figure 1. Geometry of the irradiated Fricke-gel sample, the shadowed area represents the acquired slice, and the internal cylinder is the region that was irradiated. 
Markus chamber. The proton irradiations generated a sharp concentration gradient along the axis of the samples. Images of these samples were acquired at high resolution $(0.3 \mathrm{~mm})$ with a $4.7 \mathrm{~T}$ Sisco-Varian MR research unit. An Inversion Recovery (IR) sequence with an echo time TE of $30 \mathrm{~ms}$ and a delay time $t_{\mathrm{D}}$ of $3 \mathrm{~s}$ was used. A second set of samples with a diameter of $7 \mathrm{~cm}$ and a height of $19 \mathrm{~cm}$ was irradiated inside a water phantom using a radiotherapy beam of $6 \mathrm{MV} X$ rays. The irradiation geometry was again coaxial. These X-ray irradiations generated smooth concentration gradients along the axis of the samples. Imaging in this case was done with a whole-body GE-Echospeed system operating at $1.5 \mathrm{~T}$. Axial slices were acquired with a thickness of $0.5 \mathrm{~cm}$ using a head coil.

A series of MR scans was performed at various time intervals producing R1 maps of the two sets of samples. The scans started $20 \mathrm{~min}$ after the end of irradiations, and each acquisition lasted $45 \mathrm{~min}$.

\section{METHODS}

Owing to the proportionality between 'relaxation rate' $R 1=1 / T 1$ and local concentrations ${ }^{2} \mathrm{C},{ }^{3} \mathrm{C}$ of ferrous and ferric ions we have, under suitable hypotheses,

$$
\Delta R 1(t)=R 1(t)-R 1_{\mathrm{b}}=(a-b)^{3} C(t),
$$

where $a$ and $b$ are, respectively, the effective and the true relaxivities of ferric and ferrous ion solutions, and $R 1_{\mathrm{b}}$ is the relaxation rate before irradiation.

It has been previously shown ${ }^{(2)}$ that, assuming that there is no diffusion along the $z$ axis, in the plane $z=0$, the concentration $C(x, y, t)=C$ $(x, y, 0, t)$ is a solution of the Fick's second law:

$$
\frac{\partial C}{\partial t}=D\left(\frac{\partial^{2} C}{\partial x^{2}}+\frac{\partial^{2} C}{\partial y^{2}}\right)(*)
$$

with Cauchy's initial condition $C\left(x, y, t_{0}\right)=C_{0}(x, y)$ and boundary reflection conditions:

$$
\begin{aligned}
\left(\frac{\partial C}{\partial x}\right)_{x=0} & =\left(\frac{\partial C}{\partial x}\right)_{x=h}=\left(\frac{\partial C}{\partial y}\right)_{y=0} \\
& =\left(\frac{\partial C}{\partial y}\right)_{y=2 r_{1}}=0, \quad \forall t
\end{aligned}
$$

where $h$ and $r_{1}$ correspond to the physical boundaries of the sample and are shown in Figure 1 and $D$ is the unknown diffusion coefficient.

Assuming that we can neglect the contribution at point $(x, y)$ of the points $(\tilde{x}, \tilde{y})$ whose distance is such that $|x-\tilde{x}| \geq 3 \sigma$ and $|y-\tilde{y}| \geq 3 \sigma$, where $\sigma=$ $\sqrt{2 D \Delta t}$ and $\Delta t=\left(t-t_{0}\right)$, in which $t_{0}$ is the time of irradiation of the gels at which the diffusion process begins, an approximate solution of $(*) \forall t \geq t_{0}$, is
Table 1. Estimated values for the diffusion coefficient for the 'Noxyl' and 'Xyl' samples by using MR data at 4.7 T.

\begin{tabular}{ll}
\hline Sample & $D\left(\mathrm{~mm}^{2} \mathrm{~h}^{-1}\right)$ \\
\hline Noxyl & $1.96 \pm 0.01$ \\
Xyl & $1.14 \pm 0.02$ \\
\hline
\end{tabular}

given by:

$$
\begin{aligned}
& C(x, y, t)=\frac{1}{4 \pi D \Delta t} \int_{m_{x}}^{M_{x}} \int_{m_{y}}^{M_{y}} C\left(\tilde{x}, \tilde{y}, t_{0}\right) \\
& \exp \left[-\frac{(x-\tilde{x})^{2}+(y-\tilde{y})^{2}}{4 D \Delta t}\right] . \\
& {\left[1+\exp \left(-\frac{x \tilde{x}}{D \Delta t}\right)+\exp \left(-\frac{(h-x)(h-\tilde{x})}{D \Delta t}\right)\right] .} \\
& {\left[1+\exp \left(-\frac{y \tilde{y}}{D \Delta t}\right)+\exp \left(-\frac{\left(2 r_{1}-y\right)\left(2 r_{1}-\tilde{y}\right)}{D \Delta t}\right)\right] d \tilde{x} d \tilde{y}}
\end{aligned}
$$

where: $m_{x}=\max (x-3 \sigma, 0), M_{x}=\min (x+3 \sigma, h)$, $m_{y}=\max (y-3 \sigma, 0)$ and $M_{y}=\min \left(y+3 \sigma, 2 r_{1}\right)$.

Using this expression for $C(x, y, t)$, an estimate of the diffusion coefficient $D$ can be obtained by solving the minimisation problem

$$
\hat{D}=\arg \min _{D} \mathcal{L}(D)
$$

with

$$
\mathcal{L}(D)=\sum_{(i, j) \in A}\left|C_{\text {theor }}(i, j, t)-C_{\text {meas }}(i, j, t)\right|^{2},
$$

where $\mathcal{A}$ is the region of a 2-D image over which large variations of ferric ion concentration are observed in points close to each other. $C_{\text {meas }}(i, j, t)$ is the measured ferric ion concentration derived from $\Delta R 1(t)$ ( $t$ time after irradiation). $C_{\text {theor }}(i, j, t)$ is the ferric ion concentration at a time $t$, calculated by discretising the approximate solution of Fick's Equation 1 with initial values $C_{\text {meas }}\left(i, j, t_{0}\right), t>t_{0}$.

The estimation of $D$ was performed with a numerical approach. First, we chose a finite number of points presenting a wide range of $D$ values. After checking that $\mathcal{L}(D)$ presents only one minimum in this range, we restricted the range around this value and used a higher resolution. The process was repeated 200 times starting from the same data and randomly altering the location of $\mathcal{A}$.

\section{RESULTS}

The mean values of the diffusion coefficients calculated for the Noxyl and Xyl gels are reported in Table 1, where the associated error is the standard deviation. These values were used to model 


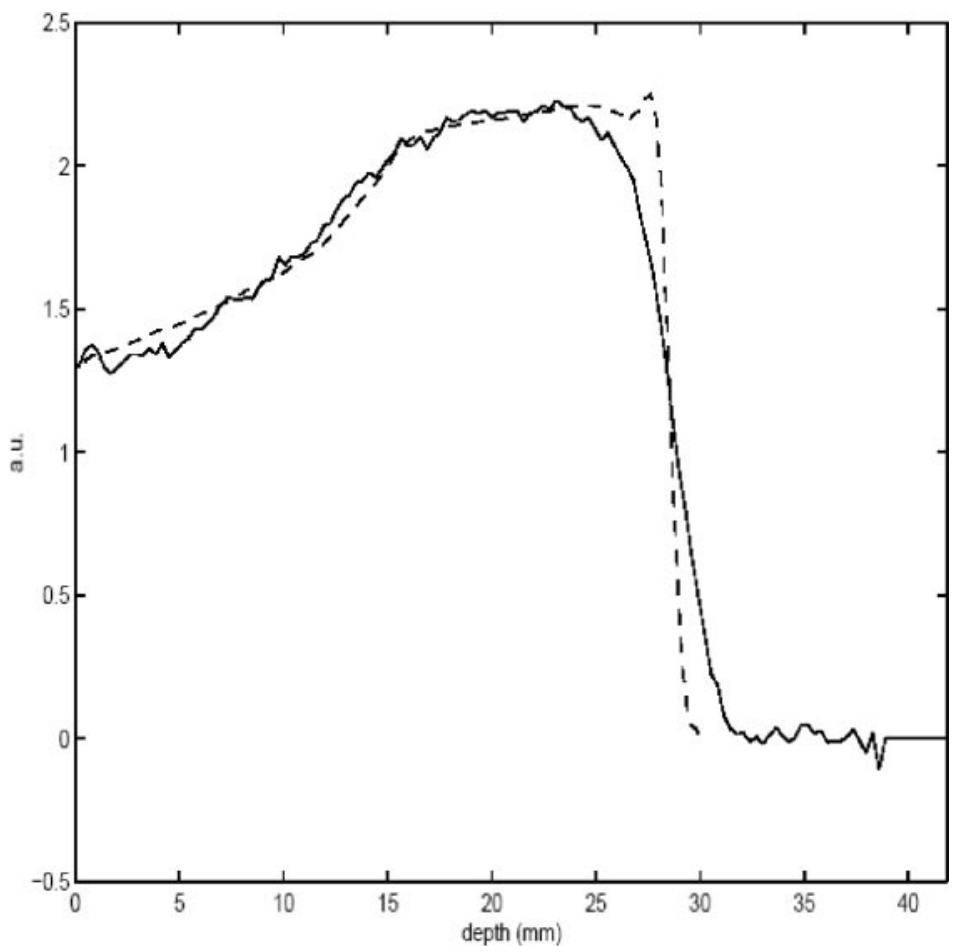

Figure 2. Comparison between dose profiles in water from MR relaxation rate variations $(\Delta R l)$ of the Fricke gels (solid line) and ion chamber measurements (dashed line).

numerically the evolution of the $\Delta R 1$ images at different times after the irradiations.

In turn, experimental $\Delta R 1$ images were generated as the difference of the $R 1(t)$ and $R 1_{\mathrm{b}}$ maps from the MR scans, using a previously reported Bayesian $\operatorname{approach}^{(3)}$. The $\Delta R 1$ images were obtained at various times following irradiation, where the time $t$ associated with each image is the average value from the end of irradiation.

Figure 2, relative to the proton irradiations, shows a comparison between Markus chamber measurements and the absorbed dose profile derived from the first $\Delta R 1$ map acquired at time $t_{1} \sim 40 \mathrm{~min}$ after the irradiation of a Xyl sample. In the smooth proximal region, preceding the Bragg peak, the agreement between ion chamber measurements and relaxation rate difference is quite good. Conversely, the diffusion effects cause the discrepancy in the sharp gradient of the distal region.

Based on our previously described model of the diffusion process and on the estimated diffusion coefficient $D$ (Table 1$)$, the $\Delta R 1\left(t_{5}\right)$ profile shown in Figure 3 was computed for a time $t_{5}=249 \mathrm{~min}$. Results from the computational modelling are clearly in agreement with the corresponding measured $\Delta R 1\left(t_{5}\right)$ map. It should be noted that it is important to include boundary reflections in the diffusion model. In their absence, a clear discrepancy arises between measured and computed data (also shown in Figure 3). Varying the spatial resolution in our data analysis had no impact on the results, this was shown by generating R1 maps at lower resolution $(1 \mathrm{~mm})$ by averaging high-resolution maps within a $3 \times 3$ local window and then performing three-pixel image-subsampling.

The Xyl and Noxyl samples irradiated with X-ray beams were characterised by smaller concentration gradients both in longitudinal and transverse directions. Dose maps based on images acquired at a spatial resolution of $1 \mathrm{~mm}$ showed negligible differences with the dose profiles from reference dosimetry, indicating that diffusion effects are much smaller. This was also confirmed by the simulations, indicating minimal changes of the dose profiles as a function of time from the irradiation.

\section{CONCLUSIONS}

Our investigations indicate that ion diffusion is not a critical issue for dose reconstruction from MRI with spatial resolution of $1 \mathrm{~mm}$ when images are taken within a few hours of the irradiation and when the 


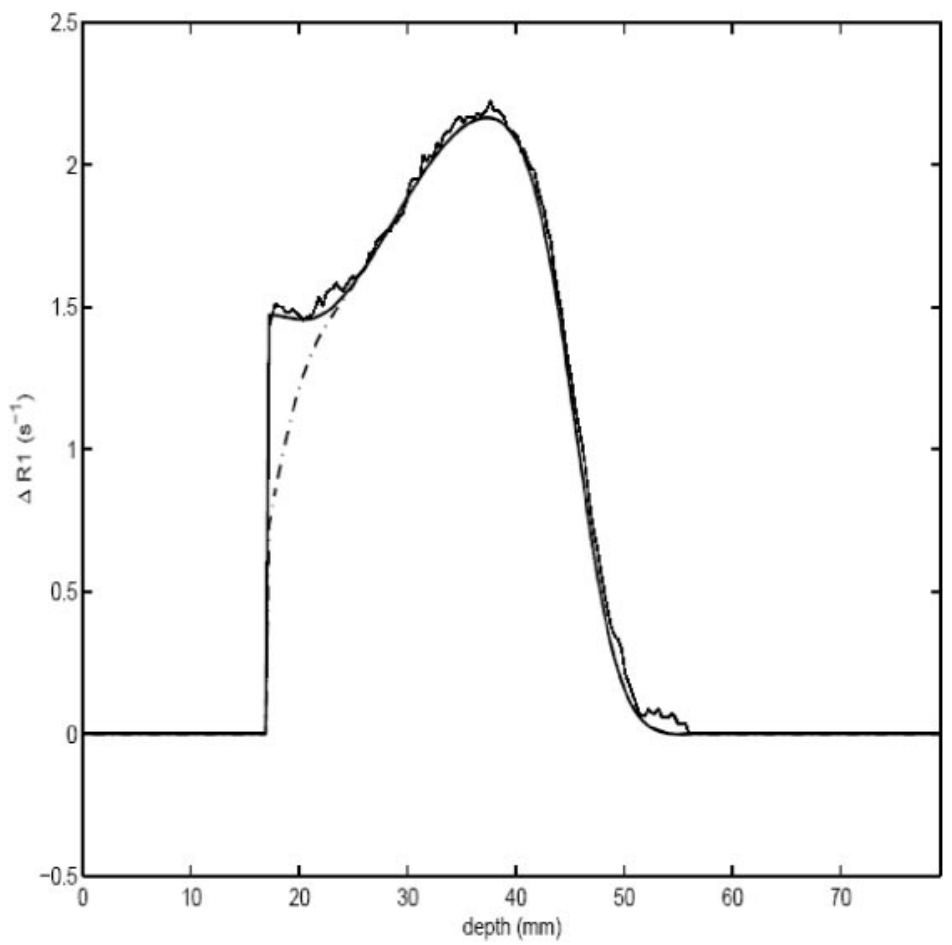

Figure 3. Comparison between dose profiles at a time $t_{5}$ based on measured $\Delta R 1\left(t_{5}\right)$ values (solid lines) and on the simulated temporal evolution of the initial $\Delta R I\left(t_{1}\right)$ image either with or without boundary reflections (bold and dotteddashed lines, respectively).

irradiation does not generate strong concentration gradients $\left(0.1 \mathrm{~s}^{-1} \mathrm{~mm}^{-1}\right.$ in $R 1$ maps), e.g. with conventional X-ray irradiations. However, when large concentration gradients are generated $\left(0.6 \mathrm{~s}^{-1} \mathrm{~mm}^{-1}\right.$ in $\left.R 1 \mathrm{maps}\right)$, e.g. following proton beam irradiations, diffusion effects are not negligible and must be taken into account. In this case, our proposed model is extremely useful, since the initial ion concentration can be accurately reconstructed based on measurements performed even several hours after irradiation.

\section{REFERENCES}

1. Appleby, A. and Leghrouz, A. Imaging of radiation dose by visible color development in ferrous-agarose-xylenol orange gels. Med. Phys. 18, 309-312 (1991).

2. Barone, P. and Sebastiani, G. Solving an inverse diffusion problem for Magnetic Resonance dosimetry by a fast regularization method. Real Time Imag. 7, 21-29 (2001).

3. de Pasquale, F. et al. Bayesian estimation of relaxation times T1 in MR images of irradiated Fricke-agarose gels. Magn. Reson. Imag. 18, 721-731 (2000). 